\title{
Full Current Statistics in Diffusive Normal-Superconductor Structures
}

\author{
W. Belzig* and Yu. V. Nazarov \\ Department of Applied Physics and Delft Institute of Microelectronics and Submicrontechnology, Delft University of Technology, \\ Lorentzweg 1, 2628 CJ Delft, The Netherlands
}

(Received 2 February 2001; published 23 July 2001)

\begin{abstract}
We study the current statistics in normal diffusive conductors in contact with a superconductor. Using an extension of the Keldysh Green's function method we are able to find the full distribution of charge transfers for all temperatures and voltages. For the non-Gaussian regime, we show that the equilibrium current fluctuations are enhanced by the presence of the superconductor. We predict an enhancement of the nonequilibrium current noise for temperatures below and voltages of the order of the Thouless energy $E_{\mathrm{Th}}=D / L^{2}$. Our calculation fully accounts for the proximity effect in the normal metal and agrees with experimental data.
\end{abstract}

DOI: 10.1103/PhysRevLett.87.067006

The electric current in conductors in general fluctuates. The full statistics of these fluctuations can be accessed in some cases [1]. However, the full statistics are not easily accessible experimentally. The current experiments mainly concentrate on noise power measurements. This has become an important tool to extract information about the relevant charge transport mechanism [2]. One can, for example, extract the effective charge of carriers responsible for the transport. Heterostructures with normal metals $(N)$ and superconductors $(S)$ were intensively investigated. Generally, the interest in these stems from the interplay between transport of doubly charged Cooper pairs and singly charged normal quasiparticles. The ratio between noise power in the superconducting state and in the normal state can roughly be interpreted as effective charge. Experimental indications of a doubled shot noise due to Andreev reflection in diffusive wires with one superconducting lead were reported in Refs. [3,4]. Other contributions report an enhancement of the current noise in SNS structures [5] with effective charges much larger than unity, possibly originating from multiple Andreev reflections.

On the theoretical side, first calculations of the noise [6,7] and the full statistics [8] for short contacts predicted an enhanced shot noise with respect to the normal state value. For certain cases, such as tunnel junctions or disordered contacts, a doubled effective charge was predicted. The drawback of these calculations is the limitation to short contacts or small energies, since fully coherent propagation of electrons and holes is assumed and the energy dependence of the scattering amplitudes has been disregarded. For the opposite regime, where coherence of electrons and holes in the normal region plays no role, a modification of the Boltzmann-Langevin approach was recently put forward [9]. Interestingly, it also gives a doubling of the shot noise in the incoherent regime. One finds similar limitations in the available calculations of the noise of $S N S$ structures [10]. It is tempting to "interpolate" between these two limits of coherent and incoherent propagation and to conclude that nothing interesting happens in the intermediate regime. We will show below that this is not the case. As
PACS numbers: 74.50. $+\mathrm{r}, 05.40 .-\mathrm{a}, 72.70 .+\mathrm{m}, 73.23 .-\mathrm{b}$

an analogy let us note the similarity to the linear conductance of a diffusive normal wire with one superconducting lead. By using the previously mentioned approaches, one finds that the conductance has exactly the same value as in the normal state independent of temperature. Only a full calculation using the Keldysh Green's function technique revealed that the conductance is significantly enhanced at energies of the order of the Thouless energy $E_{\mathrm{Th}}$ [11], displaying a reentrant behavior. This behavior of the conductance has been experimentally verified. At present no calculation of current noise is available, which fully accounts for the peculiarities of the proximity effect.

Another recent development is to study theoretically the full statistical properties of current fluctuations. The method of choice to do so is the so-called full counting statistics, pioneered in Ref. [1]. Introducing a counting field $\chi$ that couples to the current operator, one can access the full current distribution. Derivatives of the current with respect to $\chi$ immediately generate all moments of the distribution. Thus, one circumvents the cumbersome calculation of all the moments.

In this Letter we introduce a theoretical method that allows us to obtain the full current statistics for a wide class of $S N$ structures. We further present concrete results for the equilibrium current distribution and the nonequilibrium current noise with a full account of the proximity effect. For simplicity, we restrict ourselves here to a diffusive wire mounted between a normal reservoir and a superconducting reservoir. First, we determine the equilibrium current distribution. It turns out that the large current fluctuations are more probable in the superconducting case. Second, we calculate the temperature and voltage-dependent current noise. We find that the noise is enhanced (similar to the conductance) at voltages of the order of and for temperatures below the Thouless energy. In the respective limits our results agree with previous calculations $[7,9]$.

Let us first present the theoretical framework. We used a recently developed extension [12,13] of the Keldysh technique to compute the statistics of our proximity structure. There it was shown that the current statistics can be 
obtained by imposing the modified boundary condition on one reservoir's $(L)$ Green's function

$$
\check{G}_{L}(\chi)=e^{(i / 2) \chi \check{\tau}_{K}} \check{G}_{L} e^{-(i / 2) \chi \check{\tau}_{K}} .
$$

Here $\check{G}_{L}$ is the standard Green's function of a reservoir and $\check{\tau}_{K}=\hat{\sigma}_{1} \bar{\tau}_{3}$ is a matrix in Keldysh(')-Nambu(') space. Statistical properties are encoded in the dependence on the counting field $\chi$. The current probability distribution is found from

$$
P(I)=\int_{-\pi}^{\pi} d \chi e^{-S(\chi)-i \chi I t / e} .
$$

Here $t$ denotes the time of observation. The action $S(\chi)$ can be found from the integration of the $\chi$-dependent current

$$
-i \frac{e}{t} \frac{\partial S(\chi)}{\partial \chi}=I(\chi)=\frac{1}{8 e} \int d E \operatorname{Tr}\left[\check{\tau}_{K} \check{I}(\chi)\right] .
$$

The spectral matrix current in a diffusive wire is given by

$$
\check{I}(\chi)=-\sigma \check{G}(\chi) \frac{\partial}{\partial x} \check{G}(\chi),
$$

where $\sigma$ is the conductivity. This matrix current has to be found by defining an appropriate circuit. The manual on how to do this can be found in [14]. Note that the only change in comparison to the calculation of the conductance is the modified boundary condition (1). All other relations defining the actual circuit remain unchanged, as long as they are respecting the full matrix structure.

We stress that our approach is not in contradiction with the general scattering matrix approach of Ref. [8]. If one knew the electron-hole scattering amplitudes for the system under consideration, and would not disregard their energy dependence, one could obtain the same result. The characterization of these amplitudes would have to be performed along the lines of Ref. [15]. We also emphasize that the calculation of the full current statistics can be done in a finer, simpler, and more compact way than a separate calculation of its second order perturbation series, i.e., the noise.

Let us now specify our system. A diffusive metal is connected to a normal terminal at one end and to a superconducting terminal at the other end. Inside the mesoscopic wire the quasiclassical transport equations are obeyed [16]. In the normal metal they read

$$
D \frac{\partial}{\partial x}\left(\breve{G}(x, \chi) \frac{\partial}{\partial x} \check{G}(x, \chi)\right)=\left[-i E \bar{\tau}_{3}, \breve{G}(x, \chi)\right] .
$$

Here $D$ is the diffusion constant and $x$ is the coordinate along the wire, which has a length $L$. Its conductance is $G_{N}=\sigma A / L$ (cross section $A$ ). At both ends the boundary conditions to reservoirs have to be supplied. At the normal end with ideal connection, the Green's function is continuous: $\breve{G}(0, \chi)=\breve{G}_{L}(\chi)$. The other end is connected to a superconducting reservoir by a contact of negligible resistance, which leads to the boundary condition
$\check{G}(L, \chi)=\check{G}_{R}$. A circuit representation of the system is depicted in the inset of Fig. 1.

In a normal reservoir (which we will consider in the rest of the paper) $\breve{G}_{L}$ is given by

$$
\begin{aligned}
\check{G}_{L} & =\left(\begin{array}{cc}
\bar{\tau}_{3} & \bar{K} \\
0 & -\bar{\tau}_{3}
\end{array}\right), \\
\bar{K} & =2\left(\begin{array}{cc}
1-2 f(E) & 0 \\
0 & 1-2 f(-E)
\end{array}\right) .
\end{aligned}
$$

The distribution function at voltage $V$ and temperature $T$ is given by $f(E)=[\exp [(E+e V) / T]+1\}^{-1}$. A superconducting reservoir at zero voltage is described by

$$
\check{G}_{R}=\left(\begin{array}{cc}
\bar{R} & (\bar{R}-\bar{A}) \tanh (E / 2 T) \\
0 & \bar{A}
\end{array}\right) .
$$

Advanced and retarded Green's functions in (7) possess the structure $\bar{R}(\bar{A})=g_{R, A} \bar{\tau}_{3}+f_{R, A} \bar{\tau}_{1}, f_{R, A}=i \Delta /[(E \pm$ $\left.i \delta)^{2}-\Delta^{2}\right]^{1 / 2}$, and $g_{R, A}$ following from the normalization condition $f^{2}+g^{2}=1$ in a standard BCS superconductor.

Let us briefly comment on the numerical procedure of the solution. It is most convenient to solve the matrix equation (5) directly. For this purpose the diffusive wire is represented by a discrete set of $n$ nodes, each represented by a Green's function $\breve{G}_{k}$ connected in a series by tunnel junctions of conductance $g=(n+1) G_{N}$ [14]. The matrix current between two neighboring nodes is then given by $\breve{I}_{k, k+1}=\frac{g}{2}\left[\breve{G}_{k}, \breve{G}_{k+1}\right]$. The right-hand side of (5) has

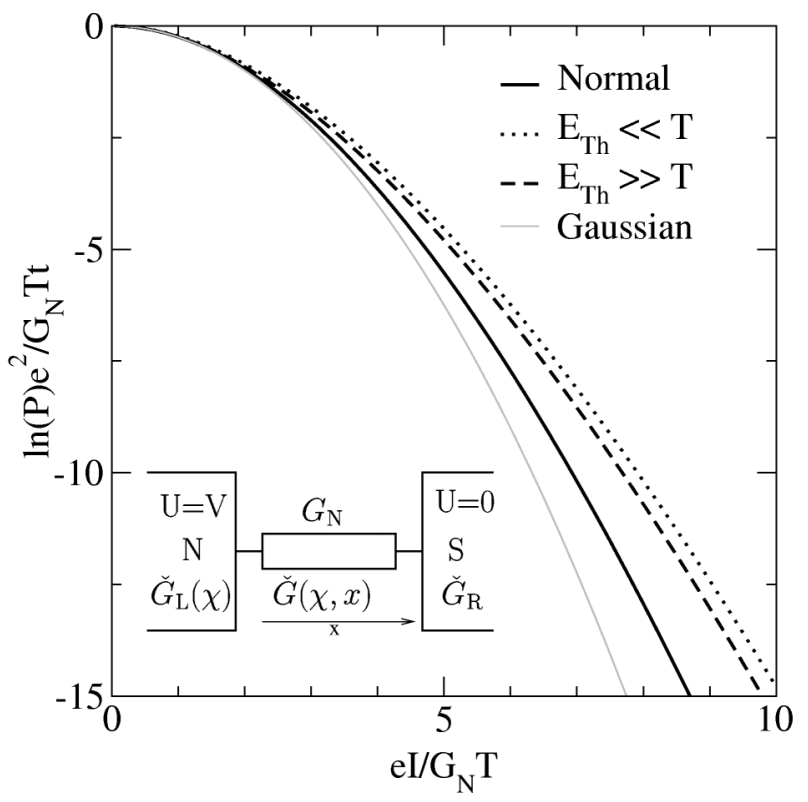

FIG. 1. Equilibrium current distribution. The case, when the superconducting terminal is normal (solid line), does not depend on the Thouless energy. In the superconducting state the distribution depends on $E_{\mathrm{Th}}$. At low temperatures (dashed curve) large fluctuations are enhanced in comparison to the normal state. This trend continues at temperatures above $E_{\mathrm{Th}}$ (dotted curve). The deviations occur in the regime of non-Gaussian fluctuations. Note that the equilibrium noise is the same in all cases. The inset depicts our model system. 
a similar form and is represented as a "decoherence" terminal [with a Green's function $\breve{G}_{\mathrm{dec}}=-i 2\left(E / n E_{\mathrm{Th}}\right) \bar{\tau}_{3}$ ] connected to each node. The matrix current conservation for node $k$ follows from discretizing Eq. (5) and reads $\left[g\left(\check{G}_{k+1}+\check{G}_{k-1}\right)+\check{G}_{\mathrm{dec}}, \check{G}_{k}\right]=0$. The resulting set of equations for the nodes' Green's functions is then solved by iteration.

As a first application, we study the distribution of current fluctuations in equilibrium. We therefore put $V=0$ and find the solution of the above equations for different values of $\chi$. We evaluate the integral over $\chi$ in (2) in the saddle point approximation, we take $\chi$ as complex and expand the exponent in (2) around the maximum. The integral then yields $P(I) \approx \exp [S(\chi)-\chi I t / e]$, which we plot implicitly as a function of $I(\chi)$. To extract generic fluctuation properties of the proximity effect we set here $\Delta \gg T, E_{\mathrm{Th}}$.

Results of this calculation are displayed in Fig. 1. The current $I$ is normalized by $G_{N} T / e$, and $\ln P(I)$ is plotted in units of $G_{N} T t / e^{2}$. The solid line shows the distribution in the normal state, which does not depend on the Thouless energy. In our units, this curve is consequently independent of temperature. In the superconducting state the Thouless energy does matter, and the distributions depend on the ratio $E_{\mathrm{Th}} / T$. We observe that large fluctuations of the current in the superconducting case are enhanced in comparison to the normal case, and in both cases are enhanced in comparison to Gaussian noise. For comparison we plotted the Gaussian distribution $\sim \exp \left(-t I^{2} / 4 G_{N} T\right)$ of the current measurements, owing to the fact that the conductance is the same in all cases. The differences between the normal and the superconducting state occur in the regime of non-Gaussian fluctuations.

Let us now turn to the nonequilibrium current noise as the second application. Previous results can be summarized in two statements. At temperatures larger than an applied voltage the wire displays the usual thermal noise $S_{I}(T \gg e V)=4 G_{N} T$ in accordance with the fluctuation-dissipation theorem. Note that in a calculation, which neglects the proximity effect, the conductance of the normal wire is equal to the normal state conductance $G_{N}$ independent of voltage or temperature. For large voltages $e V \gg T$, on the other hand, the shot noise $S_{I}(T \ll V)=$ $(4 / 3) e G_{N} V$ is doubled in comparison to the normal state value $(2 / 3) e G_{N} V$. As discussed previously these results do not depend on coherence between electrons and holes. Consequently the Thouless energy plays no role. However, this cannot be true, since the conductance is enhanced by approximately $10 \%$ at energies of the order of $E_{\mathrm{Th}}$. In the following we will show that the proximity effect indeed changes the nonequilibrium noise of the system.

Because of its importance in the following discussion, we have depicted the differential resistance of the proximity wire in the inset of Fig. 2. It displays the famous reentrance behavior [11]; i.e., starting from the normal state resistance at zero voltage a minimum at energies of

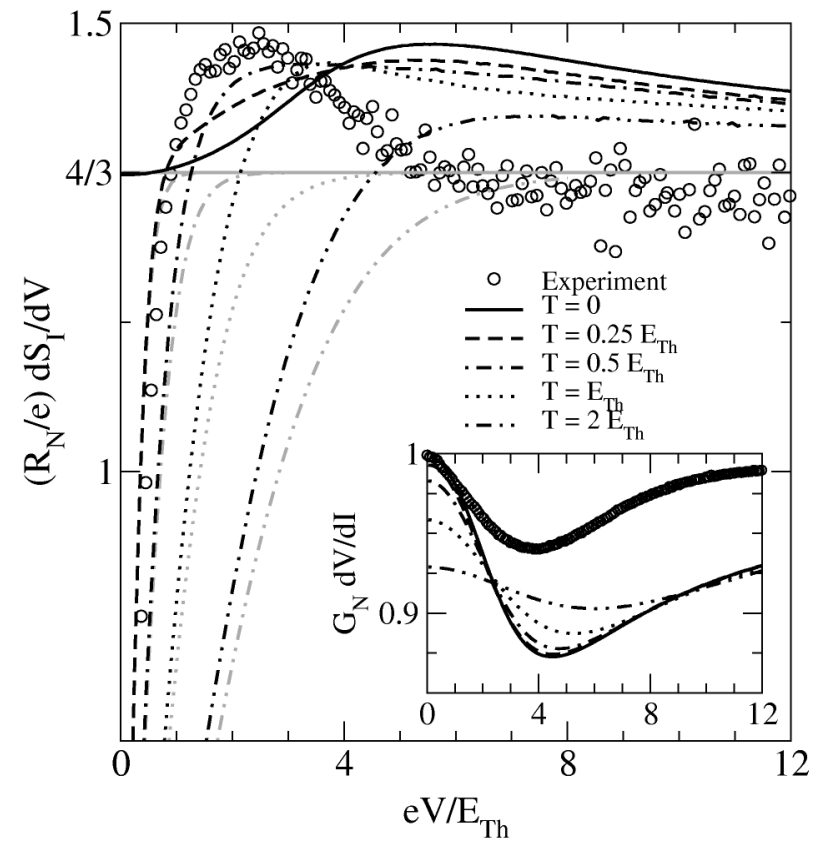

FIG. 2. Differential noise and conductance. Temperature and voltages are in units of $E_{\mathrm{Th}}$. The main plot shows our results for the differential noise together with experimental data from Kozhevnikov et al. [4] and the result of the Boltzmann-Langevin approach (grey lines). The inset shows the differential resistance. Line styles and horizontal axis correspond to the main plot, the small circles showing the corresponding experimental data of Kozhevnikov et al. [4]. At zero temperature, both differential noise and resistance show a reentrant behavior, featuring a distinct bump at voltages of the order of $\approx 5 E_{\mathrm{Th}}$. A finite temperature tends to smear this signature of the proximity effect, but an enhancement above the Boltzmann-Langevin result is still clearly visible. Comparison with the experimental data [17] shows a good qualitative agreement for $T \approx 0.25 E_{\mathrm{Th}}$, which corresponds to the experimental value. Note that our results have no adjustable parameter.

the order of several $E_{\mathrm{Th}}$ occurs. Above the minimum it decays slowly to the normal state value as $\sim\left(E_{\mathrm{Th}} / E\right)^{1 / 2}$. Therefore, the Thouless energy is the central quantity in the physics of the proximity effect. The disagreement of the theory and the experimental data from Kozhevnikov et al. [4] may possibly result from heating effects, not accounted for in the theoretical calculation.

Using the method developed in $[12,13]$, we are able to take the coherence between electrons and holes fully into account. We have to solve the Usadel equation (5), taking into account the boundary condition (1). Then, the current noise power is given by

$$
S_{I}=\left.2 e i \frac{\partial I(\chi)}{\partial \chi}\right|_{\chi=0} .
$$

There are two ways to attack this problem. One way to determine the noise is to expand the Green's functions and Eq. (5) to first order in $\chi$ and thus to obtain an equation for the noise. The other way is to solve the full matrix equations and perform the differentiation in (8) numerically. 
Below we will use the second way to find the nonequilibrium noise. Nevertheless, let us sketch the derivation of an equation for the noise. We define $\breve{G}(x, \chi)=\check{G}_{0}(x)-$ $i(\chi / 2) \breve{G}_{1}(x)$ and $\breve{I}(x, \chi)=\breve{I}_{0}(x)-i(\chi / 2) \breve{I}_{1}(x)$. As result, we find

$$
\check{I}_{1}(x)=-\sigma\left(\check{G}_{0}(x) \frac{\partial}{\partial x} \check{G}_{1}(x)+\check{G}_{1}(x) \frac{\partial}{\partial x} \check{G}_{0}(x)\right),
$$$$
\frac{D}{\sigma} \frac{\partial}{\partial x} \check{I}_{1}(x)=\left[-i E \bar{\tau}_{3}, \check{G}_{1}(x)\right] \text {. }
$$

From this equation the generalization of the BoltzmannLangevin equation to superconductors can, in principle, be derived. The boundary conditions at the reservoirs read $\check{G}_{1}(0)=\left[\check{\tau}_{K}, \check{G}_{L}\right]$ at the left end and $\check{G}_{1}(L)=0$ at the right end. Finally the noise is $S_{I}=-e \int d E \operatorname{Tr} \check{\tau}_{K} \check{I}_{1}(x)$. By taking the trace of Eq. (9) multiplied with $\check{\tau}_{K}$, it follows that it does not matter where the noise is evaluated, which is as it should be.

We now turn to concrete results for the noise power. The influence of the proximity effect is most easily seen in the differential noise $d S / d V$. Numerical results for different temperatures are displayed in Fig. 2. The inset shows the differential conductance for the same parameters. The differential noise shows a remarkable enhancement at energies of the order of the Thouless energy. Following a linear increase at low voltage the differential noise overshoots the doubled normal differential noise, which is recovered at large voltages. The maximal differential noise occurs, if the voltage is of the order of $\approx 4 E_{\mathrm{Th}}$. The largest enhancement is found for $T \ll E_{\mathrm{Th}}$ and is roughly $10 \%$. At higher temperature the differential noise approaches the Boltzmann-Langevin result [9], shown as grey lines. At zero temperature the reentrant behavior makes the connection to the result obtained within random matrix theory [7]. The nontrivial behavior in the regime between these two approaches shows the importance of phase coherence.

We can compare our results with experimental data on the noise power obtained by Kozhevnikov et al. [4,17]. The experimental parameters correspond to $T / E_{\mathrm{Th}}=0.2$. Given that our approach contains no adjustable parameters, the agreement is very good. A possible explanation for the difference between theory and experiment is that heating effects in the experiments may be important. These have been completely disregarded in the theoretical calculation. This would also explain the smaller reentrance of the differential resistance seen in the experiment. Note, however, that the energy scale, at which the influence of the proximity effect is seen, is unchanged.

In conclusion, we have developed a method to calculate statistical properties (with emphasis on the current noise power) of normal-metal-superconductor heterostructures. The method is embedded in a matrix circuit theory [14], which allows one to find the full current statistics of a wide class of systems. We applied the method to a normal diffusive wire with one normal and one superconducting reservoir. In equilibrium it turns out that the current fluctuation distribution differs from that of a purely normal system in the non-Gaussian regime. Large fluctuations of the current are enhanced by the proximity effect. For temperatures below $E_{\mathrm{Th}}$ we found that the nonequilibrium current noise shows a reentrant behavior with a maximum for voltages of the order of $E_{\mathrm{Th}}$. This is in qualitative agreement with experiment [4].

We thank A. A. Kozhevnikov and R. J. Schoelkopf for discussions and for providing us with the original data from Ref. [4]. W. B. was financially supported by the "Stichting voor Fundamenteel Onderzoek der Materie" (FOM) and the "Alexander von Humboldt-Stiftung".

*Present address: Institute of Physics and Astronomy, University of Basel, Klingelbergstrasse 82, 4056 Basel, Switzerland.

[1] L. S. Levitov, H. W. Lee, and G. B. Lesovik, J. Math. Phys. 37, 10 (1996).

[2] Ya. M. Blanter and M. Büttiker, Phys. Rep. 336, 1 (2000).

[3] X. Jehl et al., Phys. Rev. Lett. 83, 1660 (1999); X. Jehl et al., Nature (London) 405, 50 (2000).

[4] A. A. Kozhevnikov, R. J. Schoelkopf, and D. E. Prober, Phys. Rev. Lett. 84, 3398 (2000).

[5] P. Dieleman et al., Phys. Rev. Lett. 79, 3486 (1997); T. Hoss et al., Phys. Rev. B 62, 4079 (2000).

[6] V. A. Khlus, Sov. Phys. JETP 66, 1243 (1987).

[7] M. J. M. de Jong and C. W. J. Beenakker, Phys. Rev. B 49, 16070 (1994).

[8] B. A. Muzykantskii and D. E. Khmelnitskii, Phys. Rev. B 50, 3982 (1994).

[9] K. E. Nagaev and M. Büttiker, Phys. Rev. B 63, 081301(R) (2001).

[10] J. C. Cuevas, A. Martín-Rodero, and A. L. Yeyati, Phys. Rev. Lett. 82, 4086 (1999); Y. Naveh and D. V. Averin ibid., 4090 (1999); E. V. Bezuglyi et al., Phys. Rev. Lett. 83, 2050 (1999).

[11] Yuli V. Nazarov and T. H. Stoof, Phys. Rev. Lett. 76, 823 (1996).

[12] Yu. V. Nazarov, Ann. Phys. (Leipzig) 8, SI-193 (1999).

[13] W. Belzig and Yu. V. Nazarov, cond-mat/0012112.

[14] Yu. V. Nazarov, Superlattices Microstruct. 25, 1221 (1999).

[15] Yu. V. Nazarov, Phys. Rev. Lett. 73, 134 (1994).

[16] G. Eilenberger, Z. Phys. 214, 195 (1968); A. I. Larkin and Yu. N. Ovchinnikov, Sov. Phys. JETP 26, 1200 (1968); K. D. Usadel, Phys. Rev. Lett. 25, 507 (1970).

[17] The difference in the plots presented in [4] stems from the fact that we plot here the bare differential current noise $d S_{I} / d V$. That was obtained from the differential effective noise temperature $d T_{N} / d V$ (plotted in [4]) by differentiating $S_{I}(V)=T_{N}(V) / R(V)$. 